Pacific Journal of Mathematic 


\title{
POLYNOMIAL CONSTRAINTS FOR FINITENESS OF SEMISIMPLE RINGS
}

\section{Mohan S. Putcha AND AdIL YaQUB}

\begin{abstract}
Suppose $R$ is an associative ring with Jacobson radical $J$. Suppose that for each sequence $x_{1}, \cdots, x_{n}$ in $R$ there exists a polynomial $p$ homogeneous (of bounded degree) in each $x_{i}$ and a monomial $w$ in the $x$ 's, in which some $x_{i}$ is missing, such that $p=w$. Then $R / J$ is finite. It is also shown that if the above polynomial $p$ is a monomial, then $R / J$ is finite and $J$ is nil of bounded index.
\end{abstract}

In a recent paper, the authors proved the following theorem: Suppose $R$ is an associative ring with Jacobson radical $J$. Suppose further, that, for all $x_{1}, \cdots, x_{n}$ in $R$, there exists a word $w\left(x_{1}, \cdots\right.$, $\left.x_{n}\right)$, depending on $x_{1}, \cdots, x_{n}$, in which at least one $x_{i}$ (i varies) is missing, and such that

$$
x_{1} \cdots x_{n}=w\left(x_{1}, \cdots, x_{n}\right) \text {. }
$$

Then $J$ is a nil ring of bounded index and $R / J$ is finite. In the present paper, we consider the structure of an associative ring $R$ which satisfies, instead of the identity (1) above, an identity of the form

$$
p\left(x_{1}, \cdots, x_{n}\right)=w\left(x_{1}, \cdots, x_{n}\right) .
$$

In particular, we take a closer look at the structure of $R$ in those cases where (i) $p\left(x_{1}, \cdots, x_{n}\right)$ is any fixed word involving each of the variables $x_{1}, \cdots, x_{n}$ at least once, or (ii) $p\left(x_{1}, \cdots, x_{n}\right)$ is a variable polynomial in $x_{1}, \cdots, x_{n}$ with integer coefficients such that each $x_{i}$ is of the same degree in each term of $p\left(x_{1}, \cdots, x_{n}\right)$, and where these degrees are bounded. We show, for example, that if $p\left(x_{1}, \cdots, x_{n}\right)$ is as in (i) above, then the Jacobson radical $J$ of $R$ is nil of bounded index and $R / J$ is finite. Moreover, we show that, if $p\left(x_{1}, \cdots, x_{n}\right)$ is as in (ii) above, then $R / J$ is still finite. We conclude by giving some examples of the rings under consideration.

In establishing the results of this paper, we use the structure theory of rings, starting with the division ring case, then the primitive ring case, followed by the semisimple ring case

1. Main results. Throughout $R$ will denote an associative ring, $Z$ will denote the ring of integers, and $n$ will denote a fixed positive integer $>1$. We now introduce the following.

Definition 1. Let $Z\left[x_{1}, \cdots, x_{n}\right]$ be the ring of polynomials in $n$ 
noncommuting indeterminates $x_{1}, \cdots, x_{n}$ over $Z$. Let $\mathscr{V}_{n}$ be the subset of $Z\left[x_{1}, \cdots, x_{n}\right]$ consisting of polynomials $p\left(x_{1} \cdots, x_{n}\right)$ such that each $x_{i}$ appears in every term in $p\left(x_{1}, \cdots, x_{n}\right)$. By a word $w\left(x_{1}, \cdots, x_{n}\right)$ we mean a product in which each factor is $x_{i}$, for some $i$. Let $X \subseteq \mathscr{Y}_{n}$. An associative ring $R$ is called an $X$-ring if, for all $a_{1}, \cdots$, $a_{n}$ in $R$, there exists a polynomial $p\left(x_{1}, \cdots, x_{n}\right)$ in $X$ and a word $w\left(x_{1}, \cdots, x_{n}\right)$ with some $x_{j}(j$ varies $)$ missing from $w\left(x_{1}, \cdots, x_{n}\right)$, such that

$$
\begin{aligned}
& p\left(a_{1}, \cdots, a_{n}\right)=w\left(a_{1}, \cdots, a_{n}\right), \text { some } a_{j} \text { missing from } \\
& w\left(a_{1}, \cdots, a_{n}\right) .
\end{aligned}
$$

A division ring (respectively, primitive ring, semisimple ring) which is also an $X$-rind is called an $X$-division ring (respectively, $X$-primitive ring, $X$-semisimple ring).

The following lemma is immediate from the definition of an $X$-ring.

Lemma 1. (a) If $X_{1} \subseteq X_{2}$, then any $X_{1}$-ring is also an $X_{2}$-ring. (b) Any subring and any homomorphic image of an $X$-ring is also an $X$-ring.

In preparation for the proofs of the main results, we first establish the following lemmas.

Lemma 2. Suppose that $X \subseteq \mathscr{V}_{n}$ and $D$ is an $X$-division ring. Then $D$ is of prime characteristic.

Proof. Suppose that the characteristic of the $X$-division ring $D$ is zero. Then $D$ contains the rationals. Suppose that $q_{1}, \cdots, q_{n}$ are the first $n$ primes. Then, by hypothesis,

$$
p\left(q_{1}, \cdots, q_{n}\right)=w\left(q_{1}, \cdots, q_{n}\right),
$$

where each term in the polynomial $p\left(x_{1}, \cdots, x_{n}\right)$ involves every $x_{i}$, while some $x_{j}$ is missing from the word $w\left(x_{1}, \cdots, x_{n}\right)$. Thus, $q_{j}$ divides the left side of (4) but $q_{j}$ does not divide the right side of (4), a contradiction. This contradiction proves the lemma.

Lemma 3. Let $R$ be a $\mathscr{V}_{n}$-primitive ring. Then $R$ is a complete matrix ring $D_{q}$ over a division ring $D$ of prime characteristic.

Proof. Let $R$ be a $\mathscr{C}_{n}$-primitive ring. Then, by Jacobson's Density Theorem [3; p. 33], either (i) $R \cong a$ complete matrix ring $D_{q}$ over a division ring $D$, or (ii) for every positive integer $l$, there 
exists a subring $S$ of $R$ such that $S$ maps homomorphically onto $D_{l}$ for some division ring $D$. We now show that (ii) never holds. For, suppose (ii) is true. Then, by taking $l=n$ in (ii) and by using Lemma $1(\mathrm{~b})$, we readily obtain

$$
D_{n} \text { is a } \mathscr{V}_{n} \text {-ring. }
$$

Now, let

$$
a_{i}=\left[\begin{array}{lllllllll}
1 & 1 & & & & & & \\
& & & & & & & & 0 \\
& & & 0 & & & & \\
& & & & & 1 & 1 & & \\
& & & & & & \cdot & 1
\end{array}\right],(i=1, \cdots, n),
$$

where $a_{i}$ is a diagonal $n \times n$ matrix over $D$ with an entry of 0 in the $(i, i)$ position and with entries of 1 elsewhere on the main diagonal. By hypothesis, there exists a polynomial $p\left(x_{1}, \cdots, x_{n}\right)$ and a word $w\left(x_{1}, \cdots, x_{n}\right)$ such that

$$
\left\{\begin{array}{l}
p\left(a_{1}, \cdots, a_{n}\right)=w\left(a_{1}, \cdots, a_{n}\right) ; \text { each } a_{i} \text { appears in every } \\
\text { term in } p\left(a_{1}, \cdots, a_{n}\right) ; \text { some } a_{j} \text { is missing from } w\left(a_{1},\right. \\
\left.\cdots, a_{n}\right) .
\end{array}\right.
$$

Keeping in mind (6), and comparing the entries in the $(j, j)$ positions of the two matrices in the equation in (7), we obtain $0=1$, a contradiction. This contradiction shows that (ii) above never holds, and hence (i) above holds always. We have thus shown that

$$
\left\{\begin{array}{l}
\text { The ground } \mathscr{N}_{n} \text {-primitive ring } R \text { is a complete matrix } \\
\text { ring } D_{q} \text { over a division ring } D .
\end{array}\right.
$$

Moreover, $D$, as a subring of $R\left(=D_{q}\right)$, is a $\mathscr{V}_{n}$-division ring (see Lemma $1(b)$ ), and hence, by Lemma $2, D$ is of prime characteristic. This proves the lemma.

Lemma 4. The direct sum of $n$ rings $R_{i}$ each of which has a unit-element is not a $\mathscr{\mathscr { C }}_{n}$-ring.

Proof. Suppose that

$$
R \cong R_{1}+\ldots+R_{n} ; \text { each } R_{i} \text { has a unit-element } .
$$

Let

$$
a_{i}=(1,1, \ldots, 1,0,1,1, \ldots, 1) ; 0 \text { is in the } i \text { th position } .
$$

An argument similar to the one given in the proof of Lemma 3 
shows that we obtain a contradiction if we assume that $R$ is a $\mathscr{\mathscr { C }}_{n}$-ring. This proves the lemma.

We are now in a position to prove the following

TheOREM 1. Let $X \subseteq \mathscr{V}_{n}$. Then every $X$-semisimple ring is finite if and only if every $X$-division ring of prime characteristic is finite.

Proof. The "only if" part of the theorem being obvious, we now proceed to prove the "if" part. Thus, suppose that

(10) Every $X$-division ring of prime characteristic is finite.

Suppose, further, that $R$ is an $X$-semisimple ring which is not finite. We shall show that this leads to a contradiction. Since $R$ is semisimple, there exist ideals $I_{\alpha}(\alpha \in \Omega)$ of $R$ such that [3; p. 14]

$$
\bigcap_{\alpha \in \Omega} I_{\alpha}=(0) \text {; each } R / I_{\alpha} \text { is primitive . }
$$

Now, by Lemma 1 and Lemma 3 , it readily follows that $R / I_{\alpha}$ is a complete matrix ring $D_{q}$ over a division ring $D$ of prime characteristic. Since $D$ is a subring of $R / I_{\alpha}\left(=D_{q}\right)$, it follows, by Lemma $1(\mathrm{~b})$, that $D$ is an $X$-division ring of prime characteristic, and hence $D$ is finite, by (10). Therefore,

$$
R / I_{\alpha}\left(=D_{q}\right) \text { is finite . }
$$

Now, choose $\alpha_{1} \in R$, and having chosen $\alpha_{1}, \cdots, \alpha_{k}$ so that

$$
\sum_{i=1}^{k} R / I_{\alpha_{i}} \cong R / \bigcap_{i=1}^{k} I_{\alpha_{i}},
$$

choose $\alpha_{k+1} \in \Omega$ such that $\bigcap_{i=1}^{k} I_{\alpha_{i}} \nsubseteq I_{\alpha_{k+1}}$. That such $\alpha_{k+1}$ can always be so chosen is proved as follows: suppose no such $\alpha_{k+1}$ exists. Then $(0)=\bigcap_{\alpha \in \Omega} I_{\alpha}=\bigcap_{i=1}^{k} I_{\alpha_{i}}$, and hence (see (12))

$$
R \cong R / \bigcap_{i=1}^{k} I_{\alpha_{i}} \cong \sum_{i=1}^{k} \cdot R / I_{\alpha_{i}}
$$

Thus, using (11), we see that $R$ is finite, a contradiction. This contradiction shows that there exists $\alpha_{k+1} \in \Omega$ such that $\bigcap_{i=1}^{k} I_{\alpha_{i}} \nsubseteq I_{\alpha_{k+1}}$. Now, as we have seen in (11), $R / I_{\alpha_{k+1}}$ is simple. Since, moreover, $\bigcap_{i=1}^{k} I_{\alpha_{i}} \nsubseteq I_{\alpha_{k+1}}$, we have $\bigcap_{i=1}^{k} I_{\alpha_{i}}+I_{\alpha_{k+1}}=R$. Hence, by applying the second isomorphism theorem, we readily verify that

$$
R / \bigcap_{i=1}^{k+1} I_{\alpha_{i}} \cong R / \bigcap_{i=1}^{k} I_{\alpha_{i}}+R / I_{\alpha_{k+1}} \cong \sum_{i=1}^{k+1} R / I_{\alpha_{i}}
$$


by (12). In particular, we have

$$
\sum_{i=1}^{n} R / I_{\alpha_{i}} \cong R / \bigcap_{i=1}^{n} R / I_{\alpha_{i}}
$$

Hence, using Lemma $1(\mathrm{~b}), \sum_{i=1}^{n} R / I_{\alpha_{i}}$ is an $X$-ring (and thus a $\mathscr{V}_{n^{-}}$ ring) also. This, however, contradicts Lemma 4 (see (11)). This contradiction shows that $R$ is finite, and the theorem is proved.

We call a field $F$ periodic if for every $x$ in $F$, we have $x^{m}=x^{n}$ for some positive integers $m, n, m \neq n$. A periodic field which is also an $X$-ring is called an $X$-periodic field. We now prove the following

THEOREM 2. Let $X \subseteq \mathscr{V}_{n}$. Suppose that there exists a fixed integer $N$ such that, for all polynomials $p\left(x_{1}, \cdots, x_{n}\right)$ in $X$, the degree in $x_{1}, \cdots, x_{n}$ of every term in $p\left(x_{1}, \cdots, x_{n}\right)$ is less than $N$. Then, every $X$-semisimple ring is finite if and only if every $X$ periodic field is finite.

Proof. The "only if" part of the theorem being obvious, we now proceed to prove the "if" part. Thus, suppose that $R$ is an $X$-semisimple ring. Now, in view of Theorem 1, it suffices to show that

Every $X$-division ring $D$ of prime characteristic is a periodic field.

Thus, suppose that $D$ is an $X$-division ring of prime characteristic $p$, and suppose $a \in D$. We first show that

$$
a \text { is algebraic over } G F(p) \text {. }
$$

Clearly, we may assume that $a \neq 0$. Now, suppose that

$$
\begin{gathered}
q_{1}, \cdots, q_{n} \text { are fixed distinct primes; each } q_{i}>N, \text { and } \\
h_{i}=\left(q_{1} \cdots q_{n}\right) / q_{i} ;(i=1, \cdots, n) .
\end{gathered}
$$

Then

$$
q_{j} \text { divides } h_{i} \text { if and only if } i \neq j \text {. }
$$

Let $b_{i}=a^{h_{i}}$. Then since $D$ is an $X$-ring there exists a polynomial $p\left(x_{1}, \cdots, x_{n}\right) \in X$, and a word $w\left(x_{1}, \cdots, x_{n}\right)$ with some $x_{k}$ missing such that

$$
p\left(b_{1}, \cdots, b_{n}\right)=w\left(b_{1}, \cdots, b_{n}\right) .
$$

Since $x_{k}$ is missing from $w\left(x_{1}, \cdots, x_{n}\right)$ we have by (17), 


$$
w\left(b_{1}, \cdots, b_{n}\right)=a^{t} ; q_{k} \mid t .
$$

Now let $d_{2 r}$ be the degree of $x_{i}$ in the $r$ th term of $p\left(x_{1}, \cdots, x_{n}\right)$. Then each $d_{\imath r}>0$ and

$$
\begin{aligned}
& p\left(b_{1}, \cdots, b_{n}\right)=\sum_{r} m_{r} a^{c} \text { where } c_{r}=\sum_{i=1}^{n} d_{i r} h_{i} \text { and } m_{r} \text { are } \\
& \text { some integers. }
\end{aligned}
$$

By hypothesis each $d_{i r}<N$ and thus, by (15), $q_{k}>d_{i r}$. We therefore have, by (17), that $q_{k} \nmid c_{r}$ for every $r$. In particular, by (19), $c_{r} \neq t$ for any $r$. We now have, by a combination of (18), (19) and (20),

$$
\sum_{r} m_{r} a^{c} r=a^{t}, c_{r} \neq t \text { for all } r \text {. }
$$

Hence $a$ is algebraic over $G F(p)$ and (14) is proved.

Now, consider the field $(G F(p))(a)$. Since, by (14), $a$ is algebraic over $G F(p)$, it is easily seen that $(G F(p))(a)$ is a finite field, and hence

$$
a^{m}=a^{n} ; m, n \text { positive integers; } m \neq n ;(a \in D) \text {. }
$$

Thus, by Jacobson's Theorem [3], $D$ is a periodic field. The theorem now follows from Theorem 1.

In preparation for the proof of the next theorem, we now introduce the following notations and lemmas.

Suppose $a$ and $b$ are positive integers, $a>b$, which are relatively prime, and suppose

$$
V_{n}=a^{n}-b^{n},(a>b \geqq 1 ;(a, b)=1) .
$$

Let $n_{1}, n_{2}, \cdots, n_{k}$ be all the distinct positive divisors of $n$ which are less than $n$. Then $V_{n}$ is divisible by $V_{n_{1}}, V_{n_{2}}, \ldots, V_{n_{k}}$. A divisor of $V_{n}$ which is relatively prime to all of the $V_{n_{i}}(i=1, \cdots, k)$ is called a primitive divisor of $V_{n}$. For example, 5 is a primitive divisor of $2^{4}-1^{4}$.

The following lemma was proved by Birkhoff and Vandiver [1];

Lemma 5. Let $n$ be a positive integer, $n \neq 2$, and let $V_{n}$ be as in (22). Then $V_{n}$ has at least one primitive divisor other than unity, with the single exception $V_{n}=2^{6}-1^{6}$.

Next we introduce the following

Notation. $Z$ will denote the set of all positive integers. Let $s \in Z^{+}$. Then,

$$
\begin{aligned}
& D(s)=\left\{m \mid m \in Z^{+}, m \text { divides } s\right\} \\
& P(s)=\{m \mid m \in D(s), m \text { is prime }\}
\end{aligned}
$$


If $S$ is any nonempty subset of $Z^{+}$, then

$$
D(S)=\bigcup_{s \in S} D(s) \text { and } P(S)=\bigcup_{s \in S} P(s) .
$$

The following lemma is an immediate consequence of Lemma 5 .

LEMmA 6. Let $p \in Z^{+}, p>1$, and let $\left\{k_{i} \mid i \in Z^{+}\right\}$be a strictly increasing sequence of positive integers such that $k_{i}$ divides $k_{i+1}$ for each $i$. Let

$$
S=\left\{p^{k_{i}}-1 \mid i \in Z^{+}\right\}
$$

Then $P(S)$ is infinite.

We are now in a position to prove the following

Theorem 3 (Principal Theorem). Let $X \subseteq \mathscr{V}_{n}$. Suppose that there exists a fixed integer $N$ such that, for all polynomials $p\left(x_{1}\right.$, $\left.\cdots, x_{n}\right)$ in $X$, the degree in $x_{1}, \cdots, x_{n}$ of every term in $p\left(x_{1}, \cdots, x_{n}\right)$ is less than N. Suppose, further, that for all polynomials $p\left(x_{1}, \cdots\right.$, $\left.x_{n}\right)$ in $X$, each $x_{i}$ is of the same degree in each term in $p\left(x_{1}, \cdots, x_{n}\right)$. Then every $X$-semisimple ring is finite.

Proof. In view of Theorem 2, it suffices to show that every $X$-periodic field $F$ is finite. Suppose not; that is, suppose that $F$ is an infinite $X$-periodic field. Then $F$ is of prime characteristic $p$, since $F$ is periodic. Moreover, the subfield $\langle x\rangle$ generated by a single element $x$ in $F$ is finite, and hence

$$
x^{p^{k}}=x \text { for some positive integer } k=k(x) \text {. }
$$

Now, for each $j \in Z^{+}$, define

$$
F_{j}=\left\{x \mid x \in F, x^{p j}=x\right\} .
$$

Then, in view of (23) and (24), we have (since if $x \in F$ satisfies (23), then $x \in F_{k}$ )

$$
\begin{aligned}
& F_{1} \subseteq F_{2} \subseteq F_{3} \subseteq \cdots ; \text { each } F_{i} \text { is a finite subfield of } F ; \\
& \bigcup_{i \in Z^{+}} F_{i}=F
\end{aligned}
$$

Now, since $F$ is infinite, we can find a subsequence of (25) such that

$$
F_{i_{1}} \subsetneq F_{i_{2}} \underset{\neq}{\subsetneq} F_{i_{3}} \subsetneq \ldots \text {, and again } \bigcup_{k \in Z^{+}} F_{\imath_{k}}=F \text {. }
$$

Moreover, the order of $F_{i_{\sigma}}=p^{k_{\sigma}}\left(\sigma \in Z^{+}\right)$. Next, let

$$
S=\left\{p^{k}-1 \mid \sigma \in Z^{+}\right\} \text {. }
$$


Then, as is well known, $k_{\sigma} \mid k_{\sigma+1}$ for each $\sigma \in Z^{+}$, and $k_{\sigma}<k_{\sigma+1}$, by (26). Henc by Lemma $6, P(S)$ is infinite, and there, therefore, exist $n$ distinct primes $q_{1}, \cdots, q_{n}$ in $P(S)$, such that

$$
q_{i}>N+p, \quad(i=1, \cdots, n) .
$$

Thus, there exists $m_{j} \in Z^{+}$such that

$$
q_{j} \in P\left(p^{k_{m_{j}}}-1\right), \quad(j=1, \cdots, n) .
$$

Now, since the nonzero elements of the field $F_{i_{m_{j}}}$ form a multiplicative group of order $p^{k_{m_{j}}}-1$, and since the prime $q_{j} \mid p^{k_{m}}-1$, it follows, by Cauchy's theorem, that there exists $a_{j} \in F_{i_{m_{j}}}(\subseteq F)$ such that

$$
\text { order of } a_{j}=q_{j} ; a_{j} \neq 0 \quad(j=1, \cdots, n) .
$$

Now, since $F$ is an $X$-ring, there exists a polynomial $p\left(x_{1}, \cdots, x_{n}\right)$ in $X$ and a word $w\left(x_{1}, \cdots, x_{n}\right)$ such that

$$
\left\{\begin{array}{l}
p\left(a_{1}, \cdots, a_{n}\right)=w\left(a_{1}, \cdots, a_{n}\right) ; \text { each } x_{i} \text { appears in every } \\
\text { term in } p\left(x_{1}, \cdots, x_{n}\right) ; \text { some } x_{j} \text { is missing from } w\left(x_{1}, \cdots, x_{n}\right) ; \\
\text { all coefficients in } p\left(x_{1}, \cdots, x_{n}\right) \text { are integers }
\end{array}\right.
$$

Moreover, recalling that $F$ is commutative, and using the hypothesis regarding the degrees of the $x_{i}$ 's in the various terms of $p\left(x_{1}, \cdots\right.$, $x_{n}$ ), we see that

$$
\left\{\begin{array}{l}
p\left(a_{1}, \cdots, a_{n}\right)=m w_{1}\left(a_{1}, \cdots, a_{n}\right) ; m \text { an integer } \\
w_{1}\left(a_{1}, \cdots, a_{n}\right) \text { a word involving every } a_{i} .
\end{array}\right.
$$

Furthermore, $m \neq 0$, since $w\left(a_{1}, \cdots, a_{n}\right) \neq 0$ (see (29), (30) and recall that each $a_{i} \neq 0$ ). Hence, by Fermat's Little Theorem (recall that $F$ is of prime characteristic $p$ ), we have

$$
m^{p-1}=1 \text {. }
$$

Now, let $c_{j}$ be the degree of $x_{j}$ in the word $w_{1}\left(x_{1}, \cdots, x_{n}\right)$. Then, by hypothesis,

$$
c_{j}<N ; \quad(j=1, \cdots, n) .
$$

Let

$$
M=\frac{q_{1} \cdots q_{n}}{q_{j}} \cdot(p-1)
$$

Then, by (29), (30),

$$
\left(m w_{1}\left(a_{1}, \cdots, a_{n}\right)\right)^{M}=\left(w\left(a_{1}, \cdots, a_{n}\right)\right)^{M} .
$$

Hence by (31), (28), (33), and the fact that $a_{j}$ is missing from the 
word $w\left(a_{1}, \cdots, a_{n}\right)$, the above equality reduces to

$$
\left(a_{j}^{c}\right)^{M}=1 \text {. }
$$

Therefore (see (28)), $q_{j}$ divides $c_{j} M$. This is absurd, however, since $q_{j}$ does not divide $c_{j}$ (recall that $q_{j}>N>c_{j}$; see (27), (32)), and $q_{j}$ does not divide $M$ (recall that $q_{j}>p$, by (27); also see (33)). This contradiction proves the theorem.

Next, we prove the following

THEOREM 4. Let $X$ be a collection of words in $\mathscr{V}_{n}$ each of which is of degree $<N$ in $x_{1}, \cdots, x_{n}$. If $R$ is an $X$-ring with Jacobson radical $J$, then $J$ is a nil ring of bounded index, and $R / J$ is finite.

Proof. Clearly, $X$ satisfies the hypotheses of Theorem 3, and hence the $X$-semisimple ring $R / J$ is finite. Now, to prove that $J$ is nil, let $a \in J$, and suppose that

$$
q_{1}, \cdots, q_{n} \text { are distinct primes, each } q_{i}>N \text {. }
$$

Let

$$
h_{i}=\frac{q_{1} \cdots q_{n}}{q_{i}} ; \quad(i=1, \cdots, n),
$$

and let

$$
b_{i}=a^{h_{i}} ; \quad(i=1, \cdots, n) .
$$

Then, since $R$ is an $X$-ring, there exist words $w_{1}\left(x_{1}, \cdots, x_{n}\right)$ and $w\left(x_{1}, \cdots, x_{n}\right)$ such that

$$
\left\{\begin{array}{l}
w_{1}\left(b_{1}, \cdots, b_{n}\right)=w\left(b_{1}, \cdots, b_{n}\right) ; w_{1}\left(x_{1}, \cdots, x_{n}\right) \in X ; \\
\text { some } x_{j} \text { is missing from } w\left(x_{1}, \cdots, x_{n}\right) .
\end{array}\right.
$$

Now, let

$$
\text { degree of } x_{i} \text { in } w_{1}\left(x_{1}, \cdots, x_{n}\right)=c_{i} ; \quad(i=1, \cdots, n) .
$$

Then, by (38), (37), (39), we obtain

$$
a^{c_{1} h_{1}+\cdots+c_{n} h_{n}}=a^{t} .
$$

Moreover, since $x_{j}$ is missing from the word $w\left(x_{1}, \cdots, x_{n}\right)$, it is easily seen (see (36)) that

$$
q_{j} \text { divides } t \text {. }
$$

On the other hand, since $q_{j}>N$, by (35), and $c_{j}<N$ (since $w_{1}\left(x_{1}\right.$, $\left.\left.\cdots, x_{n}\right) \in X\right), q_{j}$ does not divide $c_{j}$. Also, by (36), $q_{j}$ does not divide 
$h_{j}$, and hence the prime $q_{j}$ does not divide $c_{j} h_{j}$. However, by (36), $q_{j}$ divides $h_{i}$ for each $i \neq j$. Therefore,

$$
q_{j} \text { does not divide } c_{1} h_{1}+\cdots+c_{n} h_{n} \text {. }
$$

Comparing (41), (42), we see that

$$
c_{1} h_{1}+\cdots+c_{n} h_{n} \neq t .
$$

Now, let $c_{1} h_{1}+\cdots+c_{n} h_{n}=l$, and let

$$
M=N h_{1}+\cdots+N h_{n} ;\left(M>l \text {, since each } c_{i}<N\right) .
$$

Then, by (40), (43), (44), it is easily seen that

$$
a^{M}=a^{s} \text {, for some positive integer } s ; s \neq M .
$$

Now, if in (45), $s<M$, by iterating in (45), we can eventually make $s>M$. We have thus shown that

$$
a^{M}=a^{s} ; s>M>0 ; M \text { fixed. }
$$

Equation (46) readily implies that a suitable power of $a$ is an idempotent element in $J$ (recall that $a$ is in $J$ ), and hence by $(46), a^{H}=0$, ( $M$ fixed). Thus $J$ is nil of bounded index, and the theorem is proved.

In view of Theorem 4, it follows that $J$ is locally nilpotent [2; p. 28].

The following corollary is an immediate consequence of Theorem 4 as well as Theorem 3 .

Corollary 1. Let $X$ consist of a single fixed word involving each of the variables $x_{1}, \cdots, x_{n}$. Then every $X$-semisimple ring is finite.

If, further, we let $X$ consist of the single fixed word $x_{1} \cdots x_{n}$, we obtain, as a further corollary of Theorem 4, the following result which has already been proved by the authors [5]:

COROLLARY 2. Let $R$ be an associative ring with Jacobson radical $J$ and with the property that, for all $x_{1}, \cdots, x_{n}$ in $R$, there exists $a$ word $w\left(x_{1}, \cdots, x_{n}\right)$ depending on $x_{1}, \cdots, x_{n}$, in which at least one $x_{i}$ ( $i$ varies) is missing, and such that $x_{1} \cdots x_{n}=w\left(x_{1}, \cdots, x_{n}\right)$. Then $J$ is a nil ring of bounded index and $R / J$ is finite.

2. Examples and remarks. In the following examples, we show that the class of $X$-rings subsumes all finite rings and all nilpotent rings. We also give an example of an $X$-ring which is neither finite 
nor nilpotent.

EXAMPLE 1. Let $R$ be any finite ring with exactly $m$ elements. Let $x_{1}, \cdots, x_{m+1}$ be any elements of $R$. Then $x_{i}=x_{j}$ for some $i>j$, and hence

$$
\begin{aligned}
x_{1} \cdots x_{m+1} & =x_{1} \cdots x_{j} \cdots x_{i-1} x_{j} x_{i+1} \cdots x_{m+1} \\
& =w\left(x_{1}, \cdots, x_{i-1}, x_{i+1}, \cdots, x_{m+1}\right) .
\end{aligned}
$$

Thus $R$ is an $X$-ring, where $X=\left\{x_{1} \cdots x_{m+1}\right\}$.

EXAmple 2. Let $R$ be any nilpotent ring, say $R^{m}=(0)$. Then, for all elements $x_{1}, \cdots, x_{m+1}$ of $R$, we have

$$
x_{1} \cdots x_{m+1}=0=x_{1} \cdots x_{m} \text {. }
$$

Thus $R$ is an $X$-ring, where $X=\left\{x_{1} \cdots x_{m+1}\right\}$.

ExAmPle 3. Let $R_{0}$ be an infinite field of characteristic 2, and let

$$
R=\left\{\left(\begin{array}{ll}
a & u \\
0 & 0
\end{array}\right) \mid a \in G F(2), \quad u \in R_{0}\right\} .
$$

Let $x_{1}, x_{2}, x_{3}$ be any elements of $R$. Then, as is readily verified,

$$
\begin{array}{lll}
x_{1} x_{2} x_{3}=x_{2} x_{3} & \text { if } & x_{1}=\left(\begin{array}{ll}
1 & u \\
0 & 0
\end{array}\right) \\
x_{1} x_{2} x_{3}=x_{1} x_{2} & \text { if } & x_{1}=\left(\begin{array}{ll}
0 & u \\
0 & 0
\end{array}\right) .
\end{array}
$$

Hence $R$ is an $X$-ring, where $X=\left\{x_{1} x_{2} x_{3}\right\}$. Observe that $R$ is neither finite nor nilpotent. In fact, $R$ is not isomorphic to any finite direct sum of finite or nilpotent rings.

Returning to our Principal Theorem (Theorem 3), we have the following

REMARK. In the proof of Theorem 3, we showed that every $X$ periodic field is finite. We claim that the group-theoretic analogue of this result is false. To see this, consider the group $Z\left(p^{\infty}\right)$, which consists of the set of all $p^{n}$ th roots of unity, where $p$ is a fixed prime and $n=0,1,2, \cdots\left[4\right.$, p. 4]. Suppose that $x_{1}, x_{2} \in Z\left(p^{\infty}\right)$. Then, for some integer $n, x_{1}, x_{2} \in Z\left(p^{n}\right)$, where $Z\left(p^{n}\right)$ is the group of all $p^{n}$ th roots of unity. Let $\sigma$ be a generator of $Z\left(p^{n}\right)$. Then

$$
x_{1}=\sigma^{r}, x_{2}=\sigma^{s} ; 1 \leqq r \leqq p^{n}, 1 \leqq s \leqq p^{n} .
$$


Now let

$$
r=r_{0} p^{i}, s=s_{0} p^{j} ;\left(r_{0}, p\right)=1,\left(s_{0}, p\right)=1,
$$

and suppose, without loss of generality, that $i \leqq j$. Since $\left(r / p^{i}, p\right)=1$, there exists a solution $x$ to

$$
\left(r / p^{i}\right) x \equiv s / p^{j} \bmod p^{n},
$$

and hence $r x p^{j-i} \equiv s \bmod p^{n}$. Thus, $r+s \equiv r\left(1+x p^{j-i}\right) \bmod p^{n}$, and hence

$$
\sigma^{r+s}=\left(\sigma^{r}\right)^{1+x p^{j-i}},
$$

since $\sigma^{p^{n}}=1$. Therefore $x_{1} x_{2}=\left(x_{1}\right)^{1+x p^{j-i}}$. Note that $Z\left(p^{\infty}\right)$ is an infinite group.

We leave as an open question whether or not Corollary 1 is true when $X$ consists of a single fixed polynomial in which each term involves every variable $x_{1}, \cdots, x_{n}$. In view of Theorem 2 , this question reduces to whether or not an $X$-periodic field is finite in this case.

\section{REFERENCES}

1. G. D. Birkhoff and H. S. Vandiver, On the integral divisors of $a^{n}-b^{n}$, Annals of Math., 5 (1903), 173-180.

2. I. N. Herstein, Theory of rings, Math. Lecture Notes, University of Chicago, Chicago, Ill. 1961.

3. N. Jacobson, Structure of rings, rev. ed., Amer. Math. Soc. Colloq. Publ., vol. 37, Amer. Math. Soc., Providence, R. I. 1964.

4. I. Kaplansky, Infinite abelian groups, Univ. of Michigan Press, Ann Arbor, Mich., 1954.

5. M. S. Putcha and A. Yaqub, Rings satisfying monomial constraints, Proc. Amer. Math. Soc., 39 (1) (1973), 10-18.

Received October 18, 1974.

UNIVERSITy of CALIForNia, Berkeley

AND

University of California, Santa Barbara 


\section{PACIFIC JOURNAL OF MATHEMATICS}

\section{EDITORS}

RICHARD ARENS (Managing Editor)

University of California

Los Angeles, California 90024

\section{J. DugundJI}

Department of Mathematics University of Southern California Los Angeles, California 90007

D. Gilbarg and J. Milgram

Stanford University

Stanford, California 94305
University of Washington Seattle, Washington 98105

\section{ASSOCIATE EDITORS}
E. F. BECKENBACH
B. H. NeumanN
F. WolF
K. YoShIDA

\section{SUPPORTING INSTITUTIONS}

\author{
UNIVERSITY OF SOUTHERN CALIFORNIA \\ STANFORD UNIVERSITY \\ UNIVERSITY OF TOKYO \\ UNIVERSITY OF UTAH \\ WASHINGTON STATE UNIVERSITY \\ UNIVERSITY OF WASHINGTON \\ $\stackrel{*}{*} \stackrel{*}{*} \stackrel{*}{ }{ }^{*}$ AMERICAN MATHEMATICAL SOCIETY
}

The Supporting Institutions listed above contribute to the cost of publication of this Journal, but they are not owners or publishers and have no responsibility for its content or policies.

Mathematical papers intended for publication in the Pacific Journal of Mathematics should be in typed form or offset-reproduced, (not dittoed), double spaced with large margins. Underline Greek letters in red, German in green, and script in blue. The first paragraph or two must be capable of being used separately as a synopsis of the entire paper. Items of the bibliography should not be cited there unless absolutely necessary, in which case they must be identified by author and Journal, rather than by item number. Manuscripts, in triplicate, may be sent to any one of the editors. Please classify according to the scheme of Math. Reviews, Index to Vol. 39. All other communications should be addressed to the managing editor, or Elaine Barth, University of California, Los Angeles, California, 90024.

The Pacific Journal of Mathematics expects the author's institution to pay page charges, and reserves the right to delay publication for nonpayment of charges in case of financial emergency.

100 reprints are provided free for each article, only if page charges have been substantially paid. Additional copies may be obtained at cost in multiples of 50 .

The Pacific Journal of Mathematics is issued monthly as of January 1966. Regular subscription rate: $\$ 72.00$ a year (6 Vols., 12 issues). Special rate: $\$ 36.00$ a year to individual members of supporting institutions.

Subscriptions, orders for back numbers, and changes of address should be sent to Pacific Journal of Mathematics, 103 Highland Boulevard, Berkeley, California, 94708.

\section{PUBLISHED BY PACIFIC JOURNAL OF MATHEMATICS, A NON-PROFIT CORPORATION}

Printed at Kokusai Bunken Insatsusha (International Academic Printing Co., Ltd.), 270, 3-chome Totsuka-cho, Shinjuku-ku, Tokyo 160, Japan.

\section{Copyright (C) 1975 by Pacific Journal of Mathematics} Manufactured and first issued in Japan 


\section{Pacific Journal of Mathematics}

\section{Vol. 57, No. $2 \quad$ February, 1975}

Norman Larrabee Alling, On Cauchy's theorem for real algebraic curves with boundary .......

Daniel D. Anderson, A remark on the lattice of ideals of a Prüfer domain ..................

Dennis Neal Barr and Peter D. Miletta, A necessary and sufficient condition for uniqueness of

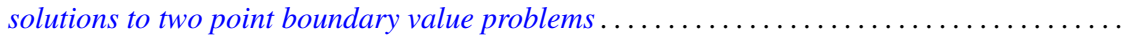

Ladislav Beran, On solvability of generalized orthomodular lattices . . . . . . . . . . ........

L. Carlitz, A three-term relation for some sums related to Dedekind sums . . . . . . . . . .....

Arthur Herbert Copeland, Jr. and Albert Oscar Shar, Images and pre-images of localization

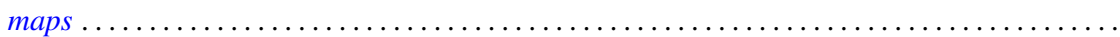

G. G. Dandapat, John L. Hunsucker and Carl Pomerance, Some new results on odd perfect

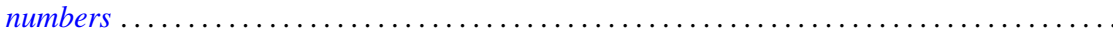

M. Edelstein and L. Keener, Characterizations of infinite-dimensional and nonreflexive

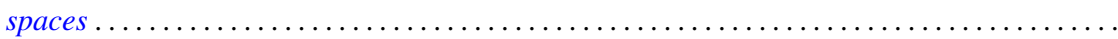

Francis James Flanigan, On Levi factors of derivation algebras and the radical embedding

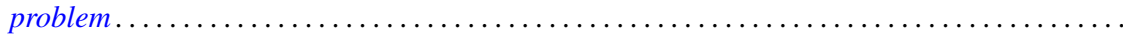

Harvey Friedman, Provable equality in primitive recursive arithmetic with and without

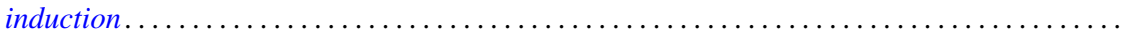

Joseph Braucher Fugate and Lee K. Mohler, The fixed point property for tree-like continua with

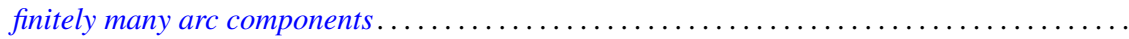

John Norman Ginsburg and Victor Harold Saks, Some applications of ultrafilters in

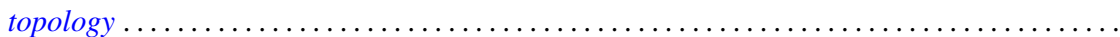

Arjun K. Gupta, Generalisation of a "square" functional equation .....................

Thomas Lee Hayden and Frank Jones Massey, Nonlinear holomorphic semigroups ..........

V. Kannan and Thekkedath Thrivikraman, Lattices of Hausdorff compactifications of a locally

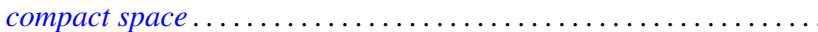

J. E. Kerlin and Wilfred Dennis Pepe, Norm decreasing homomorphisms between group

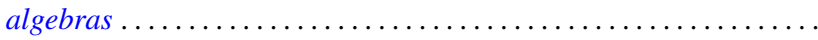

Young K. Kwon, Behavior of $\Phi$-bounded harmonic functions at the Wiener boundary ...

Richard Arthur Levaro, Projective quasi-coherent sheaves of modules .

Chung Lin, Rearranging Fourier transforms on groups...........................

David Lowell Lovelady, An asymptotic analysis of an odd order linear differential equation . . 4475

Jerry Malzan, On groups with a single involution .......................... 481

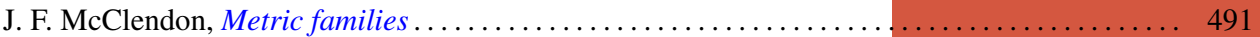

Carl Pomerance, On multiply perfect numbers with a special property .

Mohan S. Putcha and Adil Mohamed Yaqub, Polynomial constraints for finiteness of semisimple rings. .

Calvin R. Putnam, Hyponormal contractions and strong power convergence . . . . . . . . . 531

Douglas Conner Ravenel, Multiplicative operations in $\mathrm{BP} * \mathrm{BP} \ldots \ldots \ldots \ldots \ldots \ldots \ldots \ldots \ldots .539$

Judith Roitman, Attaining the spread at cardinals which are not strong limits . . . . . . . . . 545

Kazuyuki Saitô, Groups of *-automorphisms and invariant maps of von Neumann algebras . . . 553

Brian Kirkwood Schmidt, Homotopy invariance of contravariant functors acting on smooth

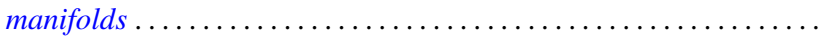

Kenneth Barry Stolarsky, The sum of the distances to $N$ points on a sphere.

Mark Lawrence Teply, Semiprime rings with the singular splitting property.

J. Pelham Thomas, Maximal connected Hausdorff spaces..............

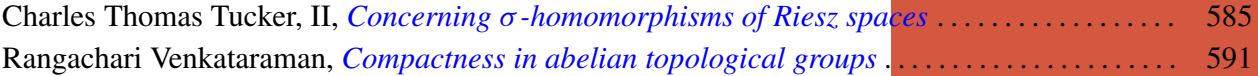

William Charles Waterhouse, Basically bounded functors and flat sheaves . . . . . . . . . . . 597

David Westreich, Bifurcation of operator equations with unbounded linearized part ......... 611

William Robin Zame, Extendibility, boundedness and sequential convergence in spaces of 